\title{
Disorder from disorder in a strongly frustrated transverse field Ising chain
}

\author{
D. J. Priour ${ }^{1}$, M. P. Gelfand ${ }^{2}$, and S. L. Sondhi ${ }^{1}$ \\ ${ }^{1}$ Department of Physics, Princeton University, Princeton, New Jersey 08544 \\ ${ }^{2}$ Department of Physics, Colorado State University, Fort Collins, Colorado 80523
}

(October 22, 2018)

\begin{abstract}
We study a one-dimensional chain of corner-sharing triangles with antiferromagnetic Ising interactions along its bonds. Classically, this system is highly frustrated with an extensive entropy at $T=0$ and exponentially decaying spin correlations. We show that the introduction of a quantum dynamics via a transverse magnetic field removes the entropy and opens a gap, but leaves the ground state disordered at all values of the transverse field, thereby providing an analog of the "disorder by disorder" scenario first proposed by Anderson and Fazekas in their search for resonating valence bond states. Our conclusion relies on exact diagonalization calculations as well as on the analysis of a 14th order series expansion about the large transverse field limit. This test suggests that the series method could be used to search for other instances of quantum disordered states in frustrated transverse field magnets in higher dimensions.
\end{abstract}

PACS numbers: 05.50.+q, 75.10.-b, 75.10.Jm, 75.30.Kz

\section{INTRODUCTION}

The study of quantum versions of classically frustrated magnets has been a subject of interest at least since the work of Anderson and Fazekas on the possibility of a quantum disordered state for the triangular lattice Heisenberg antiferromagnet 1 Following their work, and especially after the early suggestion of Anderson that the cuprate superconductors derive their special properties from the proximity of a spin liquid state 3 there has been a considerable amount of work on quantum Heisenbern models on various frustrated lattices such as the kagomé.

One can think of quantum Heisenberg models as (classical) Ising models perturbed by a transverse (XY) exchange - indeed, this was the strategy followed by Anderson and Fazekas in their analysis of the triangular lattice problem. Phrased in this fashion, the problem becomes one of the effects of introducing a quantum dynamics into a highly degenerate ground state manifold of the Ising system - a procedure of evident interest on account of the singular effects of any perturbation. The simplest instance of this more general problem is the introduction of a transverse magnetic field, which has been used in several contexts previously to generate the simplest quantum statistical mechanics; for a review see Ref. A which also reviews some work on some one-dimensional systems with competing interactions.

A number of geometrically frustrated quantum Ising systems have been studied recently by Moessner, Chandra and one of the present authors who have reported instances of "order by disorder" 5 in which a non-trivial ordering pattern is selected by the quantum fluctuations as well as one of "disorder by disorder" (on the kagomé lattice) in which the Anderson-Fazekas scenario of a disordered quantum state constructed out of a disordered classical manifold is realized. 6 In this paper, we report studies of a one-dimensional, geometrically frustrated chain which also exhibits disorder by disorder. This is interesting in itself; additionally, through a technical advance, we were able to provide strong evidence for the quantum disordered state by means of a strong coupling perturbation expansion, which we anticipate will be a useful technique in studying higher-dimensional frustrated quantum Ising systems.

\section{THE MODEL AND ITS POSSIBLE ORDERING}

We consider the Hamiltonian

$$
H=J \sum_{\langle i j\rangle} \sigma_{i}^{z} \sigma_{j}^{z}+\Gamma \sum_{i} \sigma_{i}^{x}
$$

where $J>0$ is the antiferromagnetic Ising exchange, the sum in the first term runs over the bonds of the triangular chain in Figure 1, $\Gamma$ is the strength of the transverse field and the $\sigma^{a}$ are the Pauli operators for $S=1 / 2$. (As the true spin operators are $S^{a}=\sigma^{a} / 2$, when $\hbar=1$, our $J$ is really one quarter the exchange and our $\Gamma$ is half the physical transverse field.)

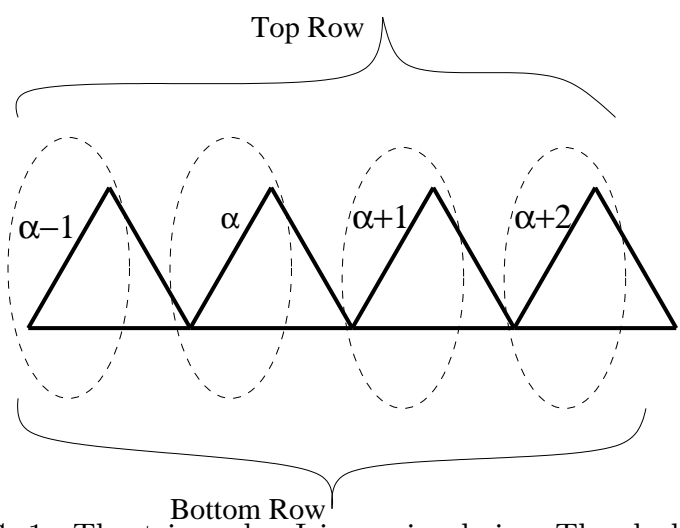

FIG. 1. The triangular Ising spin chain. The dashed ellipses enclose unit cells. 


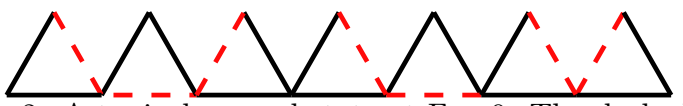

FIG. 2. A typical ground state at $\bar{\Gamma}=0$. The dashed lines represent frustrated or "bad" bonds, of which there is exactly one per triangle.

The ground states of the system without the transverse field can be obtained by minimizing the energy of each triangle separately and this in turn requires that we choose the one bond in each that will remain unsatisfied (the "bad" bond). The choice of the bad bonds is totally independent from triangle to triangle (the system is a bad bond paramagnet); a typical state is exhibited in Fig 2 . With free boundary conditions, the number of such states is thus $2 \times 3^{N}$ where $N$ is the number of triangles and the extra factor of 2 accounts for the two Ising reversed states that give rise to the same bond configuration. This extensive entropy is accompanied by short-ranged correlations and we find that the spin-spin correlation function averaged over the ground state manifold takes the form

$$
\begin{aligned}
& \left\langle S_{\alpha t}^{z} S_{\beta t}^{z}\right\rangle=\left\langle S_{\alpha b}^{z} S_{\beta t}^{z}\right\rangle=(-1 / 3)^{\beta-\alpha+1} \\
& \left\langle S_{\alpha b}^{z} S_{\beta b}^{z}\right\rangle=\left\langle S_{\alpha t}^{z} S_{\beta b}^{z}\right\rangle=(-1 / 3)^{\beta-\alpha}
\end{aligned}
$$

where $\alpha t$ and $\alpha b$ denote the top and bottom spins in unit cell $\alpha$. Due to the lack of inversion symmetry in our choice of unit cell, these forms hold for $\beta>\alpha$; they can be extended to cover $\beta \leq \alpha$ by inspecting the lattice and noting its symmetries. Evidently, the system is disordered at all temperatures.

We turn now to the quantum problem posed by the inclusion of the transverse field.

\section{A. Analysis at $\Gamma \ll J$}

At $T=0$, an infinitesimally small $\Gamma$ is a singular perturbation which will lift the macroscopic degeneracy of the classical problem. Following Ref. 6, we will attempt to identify the physics of this regime variationally. To this end we identify the configuration (unique, up to global symmetry operations) that maximizes the number of "flippable" spins, which is shown in Figure 3. A flippable spin in a given configuration is one which can be reversed without violating the ground state constraint. As a flippable spin can be polarized along $\hat{x}$ at no cost in exchange, we expect a state that maximizes their number to be especially favored by the transverse field. As not all flippable spins are independently flippable, we have two options. We may construct the staggered state in which we polarize the maximal set of independently flippable spins along the top row,

$$
|s\rangle=\left(\otimes_{\text {top }}|x-\rangle\right)|\uparrow \downarrow \uparrow \downarrow \uparrow \downarrow \cdots\rangle_{\text {bottom }} ;
$$

here $|x-\rangle$ is the state with a spin pointing down along the $\mathrm{x}$ axis. Alternatively we may construct the uniform state in which we polarize all the flippable spins but correct for the conflicts by projecting onto the ground state manifold by the action of the projector $P$ which eliminates those configurations that do not have exactly one bad bond per triangle:

$$
\begin{aligned}
|u\rangle=P\left\{\left(\otimes_{\text {top }}|x-\rangle\right)\right. & \\
& \left.\otimes(|\uparrow\rangle \otimes|x-\rangle \otimes|\uparrow\rangle \otimes|x-\rangle \otimes|\uparrow\rangle \cdots)_{\text {bottom }}\right\} .
\end{aligned}
$$

The uniform state inherits all the symmetries of the maximally flippable configuration but the staggered state does not. For the wave functions as written, we can readily evaluate the energies and we find that the staggered state has the same energy $-(\Gamma+J) / 2$ per site as the uniform state. We can also write down the magnetization (up to translations and Ising reversal) in the staggered state:

$$
\left\langle\sigma^{z}(\alpha)\right\rangle=m(\alpha)= \begin{cases}0 & \text { top } \\ \left(-\frac{1}{2}\right)^{\alpha} & \text { bottom }\end{cases}
$$

and the uniform state

$$
m(\alpha)= \begin{cases}-\frac{1}{10} & \text { top } \\ \frac{1}{10}+\left(-\frac{2}{5}\right)^{\alpha} & \text { bottom } .\end{cases}
$$

Needless to say, this analysis being purely variational is only indicative of what kind of "order from disorder" the system might exhibit at $\Gamma \ll J$. At issue is whether the connectivity of the set of configurations is too high (e.g. as reflected in the disorder in the classical problem) to permit a localization of the ground state wavefunction near the maximally flippable configuration. As advertised we will, instead, find that the system remains disordered.

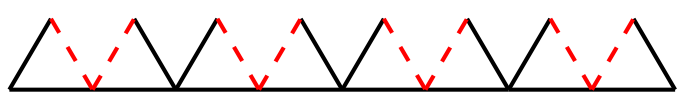

FIG. 3. The maximally flippable configuration. All sites along the top row and half of those along the bottom row are flippable.

\section{B. Analysis at $\Gamma \gg J$}

One of the attractive features of transverse field Ising models, emphasized in Ref. 6, is that they exhibit a gapped, paramagnetic phase at $\Gamma \gg J$. This enables a natural perturbative expansion for the problem, in which we perturb in the Ising exchange about the purely transverse field paramagnet. A second attractive feature exhibited by the well known Trotter-Suzuki procedure is that the quantum partition function (with the transverse field present) has the form of the classical partition function of copies of the chain (now without the transverse field) coupled ferromagnetically in the imaginary 
time direction which can then be analyzed from its "high temperature" phase via the standard Landau-GinzburgWilson (LGW) procedure. $\mathrm{It}$ is not difficult to show that these are attempts to search for the same instability. We will pursue the perturbative expansion a bit later; here, we will attempt to identify the leading instability from the paramagnetic phase by the LGW step of diagonalizing the bond (adjacency) matrix. (The equivalence to the first step in the perturbation expansion will be evident when we come to it.)

As the ferromagnetic interaction in the imaginary time direction is trivially incorporated by including a quadratic dispersion about $k_{\tau}=0$, we focus on the problem in the chains. To diagonalize the quadratic form,

$$
\mathcal{H}=\sum_{\langle i j\rangle} S_{i} S_{j}
$$

we introduce Fourier variables with respect to the unit cell location,

$$
\begin{aligned}
& S_{\alpha t}=\sum_{k} e^{i k \alpha} S_{k t} \\
& S_{\alpha b}=\sum_{k} e^{i k \alpha} S_{k b} .
\end{aligned}
$$

and find that the Hamiltonian takes the form

$$
\mathcal{H}=\sum_{k>0}\left[\begin{array}{ll}
S_{k t}^{*} & S_{k b}^{*}
\end{array}\right]\left[\begin{array}{lr}
0 & \left(1+e^{i k}\right) \\
\left(1+e^{-i k}\right) & 2 \cos k
\end{array}\right]\left[\begin{array}{l}
S_{k t} \\
S_{k b}
\end{array}\right] .
$$

Further diagonalization yields the eigenvalues

$$
\lambda_{ \pm}=(\varepsilon-1) \pm \sqrt{\varepsilon^{2}+1}
$$

where we have defined $\varepsilon=1+\cos k$. Evidently, $\lambda_{-}$is minimized when one has $\varepsilon=0 \rightarrow k=\pi$. The corresponding eigenvector is

$$
\vec{v}_{\text {min }}=\left[\begin{array}{l}
0 \\
1
\end{array}\right]
$$

Hence, the spatial dependence of the lowest eigenmode, which is the candidate for ordering, is

$$
\phi_{\pi}=\cos \pi \alpha\left[\begin{array}{l}
0 \\
1
\end{array}\right]=(-1)^{\alpha}\left[\begin{array}{l}
0 \\
1
\end{array}\right] \text {. }
$$

Thus, as $\Gamma / J$ is lowered-which corresponds to lowering the temperature in the classical representation - the LGW analysis suggests a transition in the $d=2$ Ising universality class 10 to a state with the top row disordered and bottom row ordered antiferromagnetically, which is the staggered state that we had constructed earlier.

\section{Mean Field Theory}

As we are interested in the suppression of ordering by fluctuations due to the frustration, it is useful to have a mean-field estimate for the location of the transition. To this end we consider the possibility of ordering into the staggered state. In this case only the spins on the bottom row see an effective field and the problem (in mean field theory) is identical to that of the purely one-dimensional transverse field Ising chain. This yields a critical coupling

$$
x_{c}=(J / \Gamma)_{c}=1 / 2
$$

and a staggered magnetization

$$
m=\sqrt{1-(2 x)^{-2}}
$$

in the ordered phase. The magnetization of the top row is always zero. Better estimates can be obtained by treating the top row in mean field theory and using the known exact results for the bottom row 11 In this fashion we obtain $x_{c}=1$ and $m \sim\left(x-x_{c}\right)^{1 / 8}$.

\section{EXACT DIAGONALIZATION}

\section{A. Modified Lanczos method}

We now turn to our numerical studies of the model, beginning with exact diagonalization of the Hamiltonian for finite lattices with periodic boundary conditions. Exact diagonalization unfortunately imposes a demand on computer memory which rises exponentially with the size of the system considered. Consequently, the systems discussed in this report are limited to at most 8 unit cells (16 spins).

In the modified Lanczos method that we apply 12 one begins with a state $|0\rangle$ expected to have nonzero overlap with the ground state of $\mathcal{H}$. For the present problem we used the trivial (fully $x$-polarized) $J=0$ ground state, which is a $q=0$ state. One generates a state orthogonal to $|0\rangle$ by acting upon $|0\rangle$ with $\mathcal{H}$ and using Gram-Schmidt orthogonalization to generate the state

$$
|1\rangle=\mathcal{H}|0\rangle-\frac{\langle 0|\mathcal{H}| 0\rangle}{\langle 0 \mid 0\rangle}
$$

One then diagonalizes $\mathcal{H}$ in the resulting two-dimensional subspace. The lowest eigenvalue is the improved estimate for the ground state energy, and the corresponding eigenvector is the improved estimate for the ground state wave function. This process is iterated to convergence.

A slight modification of the procedure used in obtaining the ground state energy and wave function can be employed to find the energy gap and first excited state wave function. One begins with an initial guess with nonzero overlap with the lowest excited state wave function and orthogonal to the ground state, and then proceeds as described above for the ground state. The initial guess for the excited state was chosen to be one of the excited states in the $J=0$ system, with a single flipped spin at a particular location. Although the state is not 
one of definite momentum, many iterations of the modified Lanczos procedure converge to a state of definite momentum in which $k=\pi$, as expected from the analysis in Section II. Although successive excited state estimates should in principle be orthogonal to the ground state, rounding errors cause an admixture with the ground state to occur at each iteration. To prevent a convergence toward the ground state, Gram-Schmidt orthogonalization is used at each step to remove any component of the ground state which may have entered. Although one does not have the exact ground state at a particular iteration, convergence can still be achieved by performing GramSchmidt orthogonalization with the most recent estimate for the ground state wave function.

\section{B. Excitation gap}

Figure 1 displays the gap to the first excited state computed for a variety of system sizes ( $N$ denoting the number of unit cells) and values of $x=J / \Gamma$. For convenience, the domain $[0, \infty]$ of $x$ is compressed into the interval $[0,1]$ via the transformation $y=x /(x+1)$. For most values of $y$, convergence of the gap with increasing system size is quite rapid, in itself an indication of the presence of a short correlation length. In fact, for $y<2 / 3$, the gaps for systems with 6 and 8 unit cells are virtually indistinguishable. In that regime, it is clear that the system remains disordered (in the same phase as the pure transverse-field model $y=0$ ). For larger $y$ the situation is less clear-cut, so we next consider a more sensitive test for the existence of a continuous phase transition. (We note that our largest system size yields a ground state energy of $-\frac{1}{2}[J+(1.11) \Gamma]$ per spin at $\Gamma \ll J, 11 \%$ lower than the energy for the variational states considered earlier.)

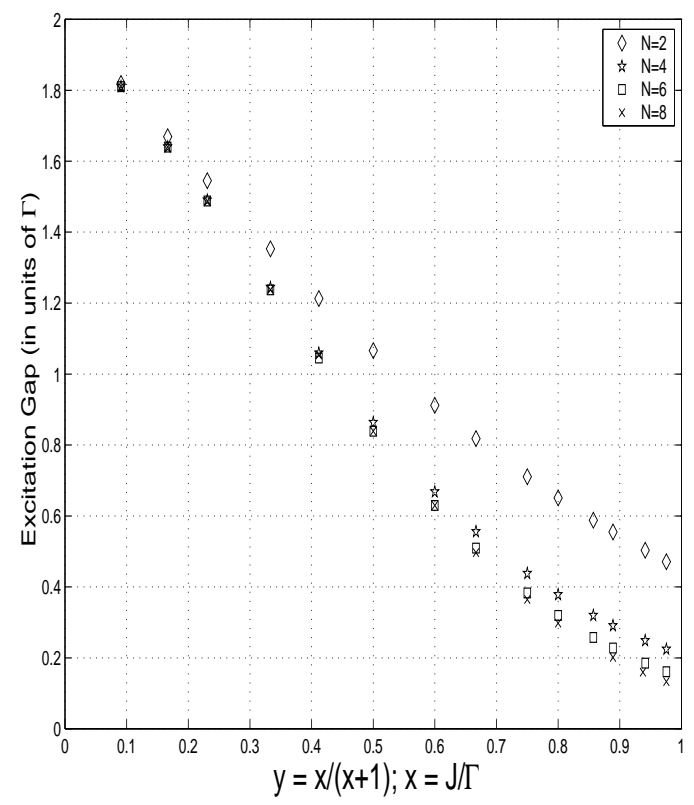

FIG. 4. Gaps obtained via exact diagonalization for $N=2,4,6,8$

\section{Cumulants}

Following Binder, 13 we examine the cumulants

$$
r=\frac{\left\langle|\Psi|^{4}\right\rangle}{\left\langle|\Psi|^{2}\right\rangle^{2}}
$$

In equation 18, $\Psi$ is the order parameter of interest. Since any ordering is expected to be accompanied by antiferromagnetic ordering of the bottom row, a sensible choice of $\Psi$ seems to be the staggered magnetic moment operator,

$$
M_{s}=\sum_{\alpha}(-1)^{\alpha} \sigma_{\alpha b}^{z}
$$

on the bottom row.

It is expected that for systems of size $L$, in the vicinity of a critical point, $r$ obeys the scaling form

$$
r=f\left(\left(x-x_{c}\right) L^{1 / \nu}\right)
$$

If a critical point exists, the cumulants computed for various system sizes should intersect at the same point for $x=x_{c}$.

Cumulants for systems containing 4, 6, and 8 unit cells are displayed in Figure 5. There is apparently no intersection of the cumulants for different system sizes for $x \leq 20$ (and, by extrapolation, for even larger values of $x)$. The behavior of the cumulants is consistent with the absence of a critical point and therefore suggests that the system is disordered for all values of $J / \Gamma$. We note that in a disordered phase with a Gaussian distributed order parameter, the cumulant will approach the value 3 . At $x=0$ the cumulant can be shown analytically to have the value $3-4 / N$ for $N$ unit cells; evidently the finite size effects are greater at large $x$, where the ground state constraint produces correlations, but even there the steady growth with system size is consistent with a thermodynamic limit value of 3 . 


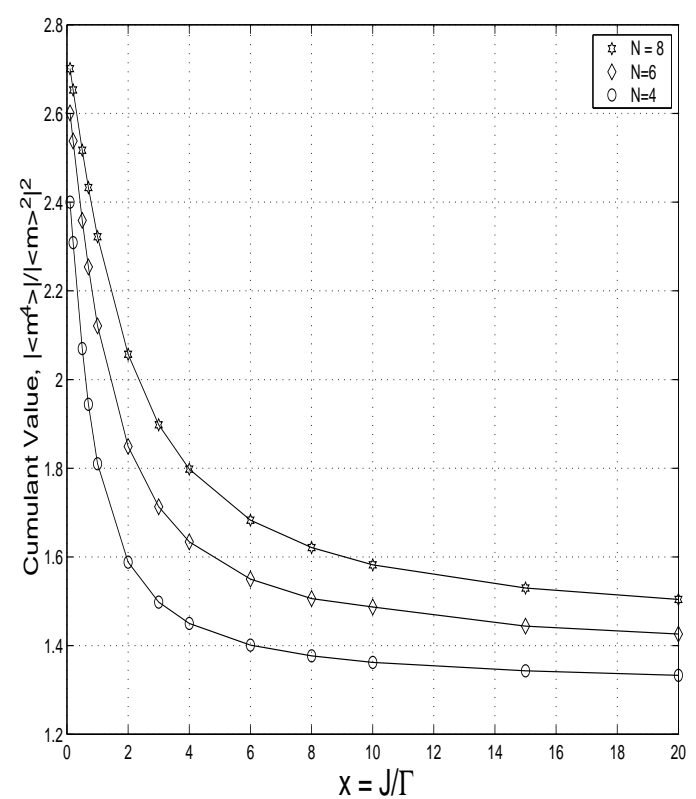

FIG. 5. Plots of the cumulant $\left\langle M_{s}^{4}\right\rangle /\left|\left\langle M_{s}^{2}\right\rangle\right|^{2}$ for triangular chains containing 4,6 , and 8 unit cells.

\section{PERTURBATION EXPANSION GENERATION AND ANALYSIS}

\section{A. Cluster expansions for the elementary excitation spectra}

A perturbation expansion for the energy gap (and the full elementary excitation spectrum, incidentally) about the $J=0$ limit was constructed to 14 th order in $J / \Gamma$ using a cluster expansion technique. The ideas underlying this calculation are set out briefly in Ref. 14, and described in more detail in Ref. 15. However, this particular calculation_is unusual (but not unique, see MüllerHartmann et al.16) in one interesting respect: it is carried out for a system with more than one branch of elementary excitations which are degenerate in the unperturbed limit, but are not degenerate by symmetry and hence are not degenerate for $J>0$. That such calculations can be done by cluster expansion methods was mentioned briefly in Ref. 15. Here we supply some further details which are important in making the connection between the the immediate product of the cluster expansion, which is a matrix containing power expansions that describe the motion of elementary excitations in real space, and the desired end product, the power series expansions for the excitation spectra in wave vector space.

For the purposes of this section we assign the sites at the base of the triangles the coordinates $(n, 0)$, and those at the tips of the triangles $\left(n+\frac{1}{2}, 1\right)$, with $n$ running over the integers. The unperturbed Hamiltonian is the transverse field $\sum_{n}\left[\sigma_{(n, 0)}^{x}+\sigma_{\left(n+\frac{1}{2}, 1\right)}^{x}\right]$ and the perturbation is the Ising exchange $\sum_{n}\left[\sigma_{(n, 0)}^{z} \sigma_{(n+1,0)}^{z}+\sigma_{(n, 0)}^{z} \sigma_{\left(n+\frac{1}{2}, 1\right)}^{z}+\right.$ $\sigma_{(n+1,0)}^{z} \sigma_{\left(n+\frac{1}{2}, 1\right)}^{z}$. The perturbation expansion is a power series in $x=J / \Gamma$.

For the unperturbed system, the ground state consists of all spins "down" (pointing along $-x$ ) and the elementary excitations are single spins which have been flipped "up". Note that there are two degenerate branches of such excitations, flat bands at $\omega=2$. (One could consider an unperturbed Hamiltonian of the more general form $\sum_{n}\left[\sigma_{(n, 0)}^{z}+\zeta \sigma_{(n, 1)}^{z}\right]$, which has the same symmetry as the triangular chain but lacks the degeneracy. For $\zeta \neq 1$, then, perturbations expansions for each of the branches of excitations could be constructed separately; however, there would be energy denominators $(1-\zeta)$ in abundance that would lead to poorly converging series for $\zeta$ near 1.)

In our calculations, with $\zeta=1$, we treat all of the elementary excitations on equal footing. We keep track of three distinct types of matrix elements in the effective Hamiltonian, those that connect two base sites (type $A$ ), those that connect two tip sites (type $B$ ), and those that connect base to tip sites (type $C$ ); let us call $t_{d}^{X}$ the matrix element (which is a series expansion in $\lambda$ ) in the effective Hamiltonian of type $X$ that couples sites at horizontal distance $d$. The effective Hamiltonian has the following structure near the diagonal

$$
\left(\begin{array}{cccccccc}
\ddots & \vdots & \vdots & \vdots & \vdots & \vdots & \vdots & \\
\cdots & t_{0}^{A} & t_{1 / 2}^{C} & t_{1}^{A} & t_{3 / 2}^{C} & t_{2}^{A} & t_{5 / 2}^{C} & \cdots \\
\cdots & t_{1 / 2}^{C} & t_{0}^{B} & t_{1 / 2}^{C} & t_{1}^{B} & t_{3 / 2}^{C} & t_{2}^{B} & \cdots \\
\cdots & t_{1}^{A} & t_{1 / 2}^{C} & t_{0}^{A} & t_{1 / 2}^{C} & t_{1}^{A} & t_{3 / 2}^{C} & \cdots \\
\cdots & t_{3 / 2}^{C} & t_{1}^{B} & t_{1 / 2}^{C} & t_{0}^{B} & t_{1 / 2}^{C} & t_{1}^{B} & \cdots \\
\cdots & t_{2}^{A} & t_{3 / 2}^{C} & t_{1}^{A} & t_{1 / 2}^{C} & t_{0}^{A} & t_{1 / 2}^{C} & \cdots \\
\cdots & t_{5 / 2}^{C} & t_{2}^{B} & t_{3 / 2}^{C} & t_{1}^{B} & t_{1 / 2}^{C} & t_{0}^{B} & \cdots \\
& \vdots & \vdots & \vdots & \vdots & \vdots & \vdots & \ddots
\end{array}\right) .
$$

This matrix acts on a column-vector describing the spinflip amplitude at each site, listed in order of its $x$ coordinate. This matrix is readily diagonalized by plane waves with a two-site basis. One obtains the two branches of the excitation spectrum in the form

$$
\epsilon_{ \pm}=\frac{1}{2}\left(F_{A}+F_{B} \pm \sqrt{\left(F_{A}-F_{B}\right)^{2}+F_{C}^{2}}\right)
$$

with

$$
F_{A}(q)=\sum_{n \geq 0} \tilde{t}_{n}^{A} \cos n q,
$$

likewise for $F_{B}(q)$, and

$$
F_{C}(q)=\sum_{n \geq 0} \tilde{t}_{(2 n+1) / 2}^{C} \cos (2 n+1) q / 2 .
$$

The relationship between the $t$ s and $\tilde{t}$ s is as follows. Take any two adjacent rows of the effective Hamiltonian, and 
count how many $t_{d}^{X}$ there are for a given $X$ and $d$ : that number is the ratio $\tilde{t}_{d}^{X} / t_{d}^{X}$. Thus $\tilde{t}_{0}^{A}=t_{0}^{A}, \tilde{t}_{1 / 2}^{C}=4 t_{1 / 2}^{C}$, and so forth. It is the $\tilde{t}_{\mathrm{s}}$ that come directly out of our computer programs and so it is convenient to express the functions that appear in Eq. (22) in those terms.

We should note that our 14 th order calculation of the excitation spectrum involved weight calculations for 1355 graphs of up to 15 spins. We did not attempt to classify the graphs topologically. The implementation of the weight calculations traded off considerable efficiency for safety (guarding against a variety of possible coding errors), and the calculations were carried out on a modest machine (333 MHz Pentium II with 256 megabytes of RAM), so it should be feasible to evaluate several more terms of the perturbation expansion if desired.

\section{B. Analysis of the energy gap series}

One is faced immediately with an interesting choice in analyzing the series for the gap, $\epsilon_{-}$. One can either extrapolate the series for $F_{A}, F_{B}$ and $F_{C}$ individually and insert the results into the formula $(22)$, or evaluate that formula for the series and extrapolate $\epsilon_{-}$directly.

However, the lowest excited state is always found at $q=\pi$, and in this case the need to choose vanishes because $F_{C}(\pi) \equiv 0$ and thus $\epsilon_{-}(\pi)=\min \left(F_{A}(\pi), F_{B}(\pi)\right)=$ $F_{A}(\pi)$. In Table [i] we display $F_{A}(\pi)$ and $F_{B}(\pi)$. The complete set of $\tilde{t}_{D}^{X}$ are available from the authors on request.

TABLE I. Coefficients of $x^{n}$ in the series $F_{A}(\pi)$ and $F_{B}(\pi)$.

\begin{tabular}{crr}
\hline \hline$n$ & \multicolumn{1}{c}{$F_{A}(\pi)$} & \multicolumn{1}{c}{$F_{B}(\pi)$} \\
\hline 0 & 2.000000000 & 2.000000000 \\
1 & -2.000000000 & 2.000000000 \\
2 & 1.000000000 & 1.000000000 \\
3 & 0.000000000 & -0.750000000 \\
4 & -0.250000000 & 0.000000000 \\
5 & 0.093750000 & -0.328125000 \\
6 & -0.144531250 & 0.785156250 \\
7 & 0.396484375 & -0.621093750 \\
8 & -0.544921875 & 0.763671875 \\
9 & 0.632377624 & -1.323387146 \\
10 & -0.908432960 & 1.746130307 \\
11 & 1.405481246 & -2.362823632 \\
12 & -2.073822043 & 3.583402837 \\
13 & 3.127851625 & -5.491168599 \\
14 & -4.983237137 & 8.550544610 \\
\hline \hline
\end{tabular}

To analyze the gap series, we applied the transformation $y=x /(x+1)$, and constructed Padé approximants to the transformed series. We use the notation $P_{M^{\prime}}^{M}$ to denote an approximant with a polynomial of order $M$ in the numerator and order $M^{\prime}$ in the denominator. Figure 6 displays several approximants which use all the terms of the series (and hence $M+M^{\prime}=14$ ).

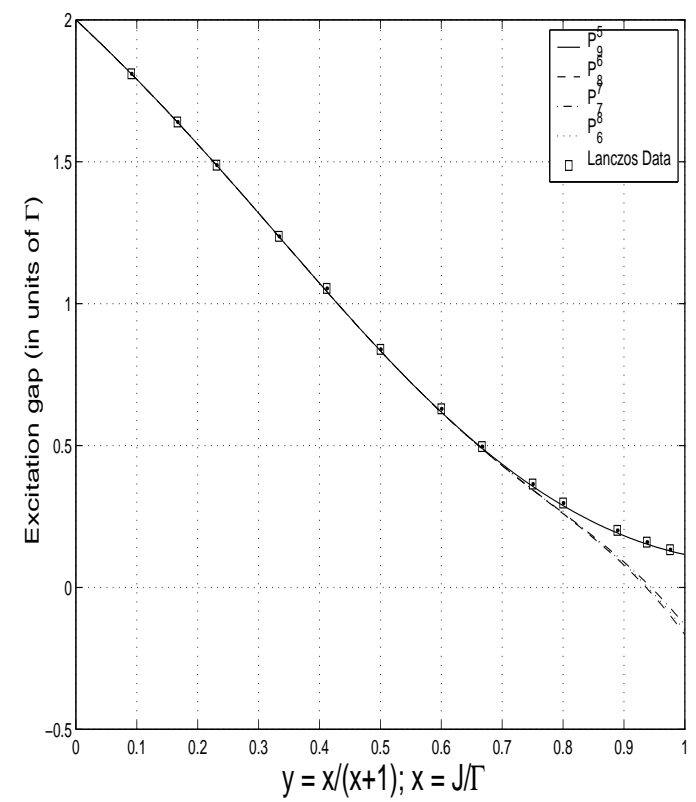

FIG. 6. Padé approximants and exact diagonalization results $(N=8)$ for the gap plotted as a function of $y=x /(x+1)$.

A salient feature of Figure 6 is the consistency of the Padé approximants over a large fraction of the domain. The approximants displayed in the plot are consistent to within $1 \%$ for $y$ as large as 0.75 . Note that this corresponds to a value of $x=3$, well in excess of the mean field estimate of $x_{c}=1 / 2$ and the $d=1$ transverse field Ising model estimate of $x_{c}=1$. Even without further analysis these indicate the disordering effects of the strong frustration in the problem.

Figure 6 also displays the excitation gap as computed via the modified Lanczos algorithm. Evident in the graph is the excellent agreement of the approximants and the exact diagonalization results over the region of consistency among the Padé approximants.

\section{Two Point Approximants and The Global Phase Portrait}

Although the region of validity of the analyzed gap series extends well into the domain of strong exchange coupling, the Padé approximants plotted in Figure 6 tell an ambiguous story. In particular, it is not clear from the displayed approximants whether or not the excitation gap vanishes for large $J / \Gamma$ and hence it is unclear 
whether the triangular chain is ordered in the $J \gg \Gamma$ limit. The reader should note though that even those Padé approximants that indicate a vanishing of the gap, do so for values of $x$ in excess of 11 .

Biasing of the Padé approximants is one means of extending the results of the gap series analysis to larger $y$ values. A simple way to bias the approximants is by means of "two-point" approximants.17 In particular, one would hope that by biasing the value of the gap at $y=1$ the resulting approximants would be accurate over the entire range $0 \leq y \leq 1$. However, since it is precisely the value of the gap at $y=1$ (let us denote that $\Delta(1)$ ) that we know least well, such a procedure appears to beg the question. What we have done, then, is to construct two-point approximants (with various orders of numerators and denominator) for a range of $\Delta(1)$ values, and observed behavior. Two of the biased approximants, $P_{9}^{5}$ and $P_{8}^{6}$, show a high degree of consistency with each other for a relatively small range of $\Delta(1)$ values. In fact, the range over which the maximum discrepancy between the two is less than $1 \%$ is confined between $\Delta(1)=.15$ and $\Delta(1)=.2$. For values of $\Delta(1)$ between .15 and $.20, P_{9}^{5}$ and $P_{8}^{6}$ are in good enough agreement that the maximum disagreement between the two over the entire $y$ domain falls below $1 \%$. Outside the $\Delta(1)$ range indicated above, the maximum disagreement between $P_{9}^{5}$ and $P_{8}^{6}$ exceeds $1 \%$. Figure 7 displays the biased approximants corresponding to the limits of $\Delta(1)$ discussed above. No matter what the choice of $\Delta(1)$, none of the other two point approximants are in agreement with each other or with $P_{9}^{5}$ and $P_{8}^{6}$ to an extent which approaches the level of agreement of $P_{9}^{5}$ and $P_{8}^{6}$ for $\Delta(1)$ values between .15 and ..20.

The biased $P_{9}^{5}$ approximants displayed in the figure are in reasonable, though not perfect agreement with the Lanzcos gap values. The approximants shown are biased at $\Delta(1)=.15$ and $\Delta(1)=.2$, the bounds of the region in which $P_{9}^{5}$ and $P_{8}^{6}$ satisfy the $1 \%$ consistency criterion discussed above. The agreement is best for small values of $y$ but deteriorates as $y$ approaches 1 . However, even at $y=1$, averaging the two approximants yields a prediction for $\Delta(1)$ of .175 , a value which differs from the Lanczos prediction of .132 (albeit without any attempt at scaling with system size) by only $30 \%$. The approximants echo the exact diagonalization results in indicating the absence of order for all values of $J / \Gamma$.

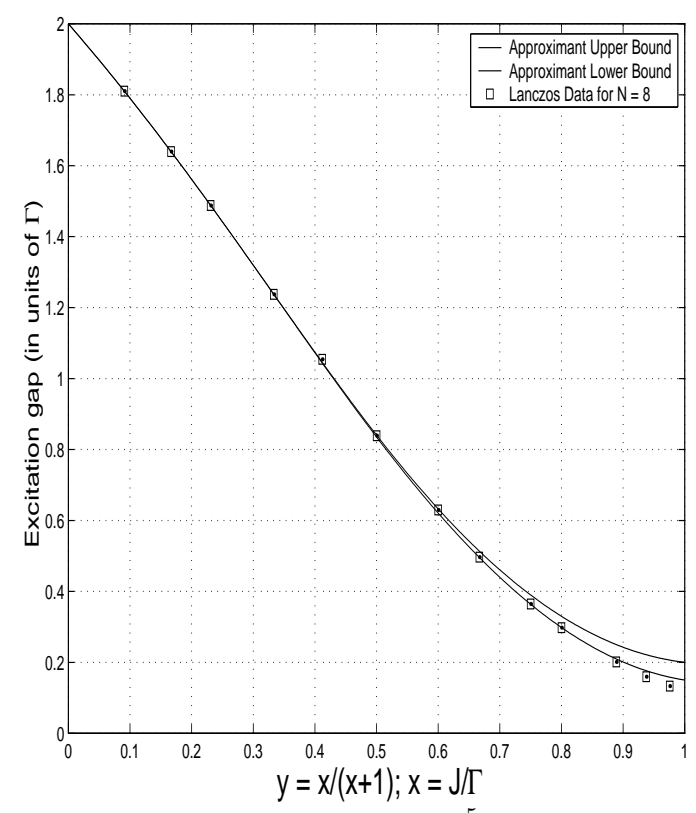

FIG. 7. Biased Padè approximant $P_{9}^{5}$ plotted at both limits of $1 \%$ consistency in juxtaposition with Lanczos results for $N=8$.

\section{SUMMARY}

Results for the energy gap and cumulants from exact diagonalization of finite systems indicate that the transverse field, triangular chain Ising model is disordered, even though several analytic approaches (from both the small and large transverse-field limits) suggest that a particular ordered phase could exist for small transverse field. We note that the lowest lying excited state is consistent with the ordering analysis, indicating that the fluctuations generated by the ground state constraint at small $\Gamma / J$ are too strong to allow order to set in. Hence we get "disorder by disorder" instead of "order by disorder". Estimates of the gap obtained by direct Padé approximants to 14th order perturbation expansions about the strong transverse field limit produce excellent results over a modest range of the Ising exchange coupling to transverse field ratio that exceeds the natural estimates for a critical value by a factor of 3 and do not indicate ordering for values of the ratio as big as 11. Globally reasonable results have been obtained by biasing the approximants and applying a consistency criterion and these indicate a lack of ordering at any value of $J / \Gamma$. This suggests that an application of this method to systems of greater experimental interest, such as the higher dimensional kagomé and pyrochlore lattices, may be a viable approach to deducing the global phase behavior of such systems. We should note that in $d>1$ we expect the series method to be increasingly competitive with exact diagonalization as the latter technique has to contend 
with much stronger finite size effects at computationally feasible system sizes.

\section{ACKNOWLEDGMENTS}

We would like to thank Roderich Moessner for collaboration in the initial stages of the project and for much useful advice in its course. We would also like to thank him for a careful reading of the manuscript. This work was supported by the National Science Foundation through grants DMR 94-57928 (MPG) and DMR 99-78074 (DJP and SLS) as well as by the Alfred P. Sloan Foundation and the David and Lucille Packard Foundation (SLS).

${ }^{1}$ P. Fazekas and P. W. Anderson, Phil. Mag. 30, 23 (1974).

${ }^{2}$ P. W. Anderson, Science 235, 1196 (1987).

${ }^{3}$ V. Elser, Phys. Rev. Lett. 62, 2405 (1989). For subsequent work see P. Sindzingre et al, cond-mat/9907220 and references therein.

${ }^{4}$ B.K. Chakrabarti, A. Dutta and P. Sen, Quantum Ising Phases and Transitions in Transverse Ising Models, (Springer-Verlag, Berlin, 1996).

${ }^{5}$ J. Villain, R. Bidaux, J. P. Carton and R. J. Conte, J. Phys. - Paris 41, 1263 (1980); E. F. Shender, Sov. Phys. JETP 56, 178 (1982).

${ }^{6}$ R. Moessner, S. L. Sondhi and P. Chandra, Phys. Rev. Lett. 84, 4457 (2000).

${ }^{7}$ With periodic boundary conditions there are $3^{N}+(-1)^{N}$ states where $N$ is the number of unit cells. Hence we have the same entropy per site in the thermodynamic limit. The reduction by a factor of two arises for the periodicity requires an odd/even number of bad bonds along the bottom row for an odd/even number of unit cells.

${ }^{8}$ M. Suzuki, Progr. Theor. Phys. 56, 1454 (1976)

${ }^{9}$ D. Blankschtein, M. Ma, A. N. Berker, G. S. Grest, C. M. Soukoulis, Phys. Rev. B 29, 5250 (1984)

10 The transverse field ferromagnetic and antiferromagnetic Ising problems are identical and in the same universality class as the $\mathrm{d}=2$ classical Ising model. As the LGW analysis indicates an antiferromagnetic ordering, the conclusion follows.

${ }^{11}$ See, for example, J. B. Kogut, Rev. Mod. Phys. 51, 659 (1979).

12 E. Dagotto, A. Moreo, Phys Rev. D 21, 865 (1985)

${ }^{13}$ K. Binder, D. W. Heermann, Monte Carlo Simulation in Statistical Physics, (Springer-verlag, Berlin, 1992).

${ }^{14}$ M. P. Gelfand, Solid State Commun. 98, 11 (1996).

${ }^{15}$ M. P. Gelfand and R. R. P. Singh, Adv. in Phys. 49, 93 (2000).

${ }^{16}$ E. Müller-Hartmann et al., Phys. Rev. Lett. 84, 1808 (2000).
${ }^{17}$ Carl M. Bender, Steven A. Orszag, Advanced Mathematical Methods for Scientists and Engineers, (McGraw - Hill, Inc., 1978). 